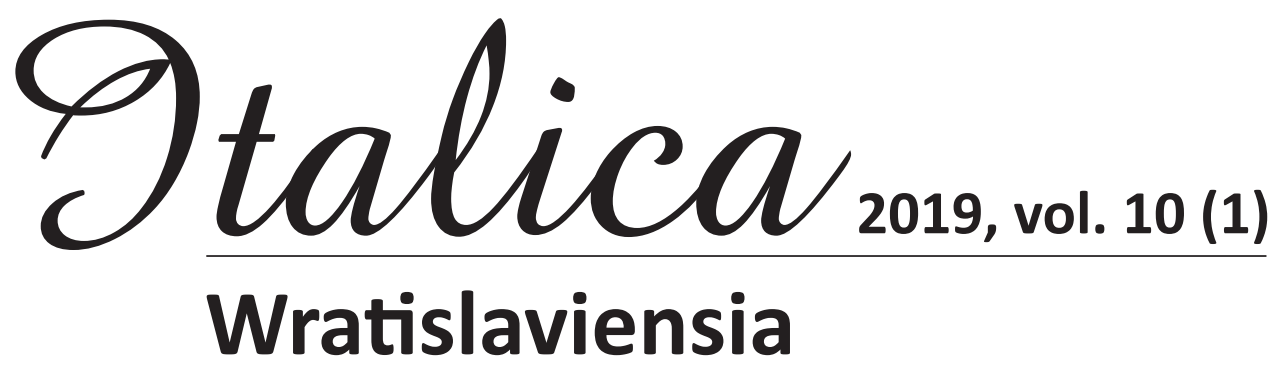

ITALIANO E LINGUE SLAVE:

PROBLEMI DI GRAMMATICA CONTRASTIVA

Edited by

Ruska Ivanovska-Naskova

Daniel Słapek 


\title{
INDICE
}

\author{
STUDI
}

\section{Helena Bažec}

Pronuncia e grafia tra L1 e L2: alcune considerazioni sugli errori ortografici di studenti slovenofoni . . . . . . . . . . . . . . . . . . . . . . . . . .

\section{Valentina Benigni \& Luisa Ruvoletto}

Asimmetrie nella codifica dell'informazione deittica: italiano $v s$ russo.......

\section{Ruska Ivanovska-Naskova}

Gli studi contrastivi dell'italianistica macedone: sviluppi recenti

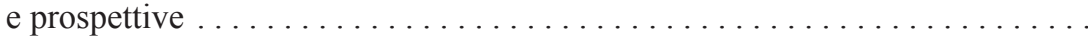

\section{Jana Kenda}

Colpa dell'interferenza: analisi contrastiva di errori negli scritti di studenti universitari slovenofoni di italiano $\ldots \ldots \ldots \ldots \ldots \ldots \ldots \ldots \ldots \ldots \ldots$

\section{Agnieszka Latos \& Aleksandra Pronińska}

Il sistema nominale italiano e polacco a confronto:

riflessioni sulle categorie grammaticali di genere e caso $\ldots \ldots \ldots \ldots \ldots$.

\section{Beata Malczewska}

Nomi e aggettivi qualificativi riguardanti la lepre nella lingua venatoria italiana e polacca.

\section{Małgorzata Nowakowska}

Come si traduce in polacco la perifrasi verbale stare per + INFINITO . . . . . . 155

Ivica Peša Matracki

Aspetti pragmatici delle strategie evidenziali in italiano e in croato ........

\section{Mirjam Premrl}

Alcune tendenze nella correlazione tra forma (in)definita dell'aggettivo sloveno e la (in)definitezza del sintagma in ottica contrastiva italo-slovena ... 


\section{Daniel Słapek}

Sulla declinazione dei cognomi italiani in polacco.............. 233

Roska Stojmenova Weber

Tradurre la lineetta singola: uno studio contrastivo macedone-italiano

\section{VARIA}

\section{Roberta lannacito-Provenzano}

Il plurilinguismo nelle opere teatrali del commediografo molisano

Antonio Angelone.

\section{Luca Palmarini}

Sul manoscritto testamentario di Bartolomeo Berrecci: uno sguardo

storico-linguistico

NOTE SUGLI AUTORI 
How to reference this article Benigni, V., \& Ruvoletto, L. (2019). Asimmetrie nella codifica dell'informazione deittica: italiano vs russo. Italica Wratislaviensia, 10(1), 31-58.

DOI: http://dx.doi.org/10.15804/IW.2019.10.1.2

\author{
Valentina Benigni \\ Università degli Studi "Roma Tre" \\ valentina.benigni@uniroma3.it, ORCID: 0000-0002-2813-3345 \\ Luisa Ruvoletto \\ Università Ca' Foscari di Venezia \\ luisa.ruvoletto@unive.it, ORCID: 0000-0002-0329-1689
}

\title{
ASIMMETRIE NELLA CODIFICA DELL'INFORMAZIONE DEITTICA: \\ ITALIANO VS RUSSO
}

\section{ASYMMETRIES IN THE ENCODING OF DEICTIC REFERENCE: ITALIAN VERSUS RUSSIAN}

\begin{abstract}
This paper aims to analyse, through a contrastive approach, the asymmetries between Italian and Russian in the encoding of deictic reference. Some complex and divergent instances of deictic anchoring are discussed in relation to the notion of deictic centre (or zero-point, in Lyons' terms). Different types of asymmetry can be identified according to structural and functional criteria: a) overcoding versus undercoding of deictic information (e.g., Italian tra and dopo versus Russian čerez); b) different internal articulation of the deictic reference (such as in the sequence of tenses, which is governed by mood and tense in Italian and solely by tense in Russian); c) the adoption of a different perspective in the deictic conceptualisation of space, time, and person (as in conversive predicates). All of these types of asymmetry seem to affect learners' acquisition of L2. For this reason, a systematic analysis of errors committed in deictic reference can be a useful tool for second-language teaching.
\end{abstract}

Keywords: deixis, deictic asymmetries, second-language acquisition, contrastive grammar, parallel corpora 


\section{OBIETTIVI E METODOLOGIA ${ }^{1}$}

Già da tempo la linguistica ha riconosciuto il ruolo dell'analisi contrastiva nell'acquisizione: l'individuazione di similitudini e divergenze tra il sistema linguistico della lingua materna e quello della lingua target permette di insistere sugli elementi che facilitano o, al contrario, rendono più difficoltoso l'apprendimento di una lingua seconda. Tradizionalmente l'approccio contrastivo si è incentrato sulla semantica lessicale e sul confronto grammaticale, dando vita a diversi filoni di ricerca, tra cui - giusto per citare i più corposi - lo studio degli elementi culturospecifici (o realia, secondo la definizione adottata nei Paesi slavi nell'ambito degli studi traduttologici), l'individuazione dei primitivi semantici (semantic primes) e l'elaborazione di un metalinguaggio (natural semantic metalanguage) come tertium comparationis negli studi di semantica contrastiva (Goddard \& Wierzbicka, 2014), la comparazione tra sistemi verbali a base aspettuale e base temporale, e l'opposizione tra strategie lessicali e grammaticali nella codifica della (in)determinatezza, su cui si basa gran parte del confronto grammaticale tra lingue slave e romanze. Più di recente anche la pragmatica interculturale è entrata nella sfera di interesse degli studi contrastivi, assumendo come oggetto di ricerca, ad esempio, il modo in cui un medesimo atto linguistico, oppure categorie pragmatiche quali la cortesia o il commitment del parlante, prendono forma in lingue e culture diverse.

Anche lo studio dei processi di significazione, $\mathrm{o}$, più genericamente, di costruzione del significato, può trarre vantaggio da un approccio contrastivo: per tale motivo abbiamo scelto di incentrare la nostra analisi sulle diverse modalità con cui italiano e russo adoperano la deissi, che costituisce lo strumento fondamentale per ancorare la dimensione linguistica alla realtà extra-linguistica. A partire dalla nostra pratica

1 Il lavoro nasce dalla stretta collaborazione tra le due autrici, i cui nomi sono riportati in ordine alfabetico. Unicamente ai fini accademici, Valentina Benigni è responsabile della stesura dei $\S \S 1,2,3,4,4.1-4.4$, mentre Luisa Ruvoletto è responsabile della stesura dei $\S \S 4.5-4.7,5,6,7,8$. 
didattica di docenti di russo lingua straniera (RKI) abbiamo osservato che molti degli errori che ricorrono nell'interlingua degli apprendenti italofoni, così come dei madrelingua russofoni che seguono i medesimi corsi per migliorare le loro competenze in italiano, sono il frutto di asimmetrie relative al modo in cui italiano e russo codificano l'informazione deittica di persona, spazio, tempo.

Obiettivo primario del lavoro pertanto è quello di individuare, descrivere e sistematizzare in base al tipo di asimmetria tali differenze, eventualmente anche in una prospettiva didattica (obiettivo secondario).

A tale proposito abbiamo seguito un approccio basato sull'uso che prevede l'adozione di una metodologia sia corpus driven che corpus based. A partire da un piccolo corpus di produzioni spontanee e semiguidate di parlanti italofoni studenti di russo e parlanti russofoni studenti di italiano raccolto all'università Roma Tre (v. § 3), sono stati estratti gli errori che a nostro parere rivelano una divergenza nel modo in cui l'informazione deittica viene organizzata nella L1 dell'apprendente rispetto alla L2 studiata. Nella seconda fase del lavoro, individuati i contesti deittici "problematici" per l'apprendente, si è deciso di verificare l'uso in corpora paralleli, così da evidenziare le differenze nel modo in cui un medesimo significato deittico viene concettualizzato in lingue differenti. I corpora paralleli utilizzati sono il corpus russo-italiano presente sul Corpus Nazionale di Lingua Russa, prevalentemente basato su testi letterari e di pubblicistica, e la risorsa Kontext, accessibile dal Corpus Nazionale di Lingua Ceca, che permette di allineare testi in diverse lingue attingendo dai corpora della famiglia InterCorp: questa risorsa, oltre a contenere alcuni testi letterari e pubblicistici tradotti in diverse lingue, si basa in larga misura sull'allineamento di sottotitoli televisivi e cinematografici. Alcuni degli esempi discussi relativi a quest'ultimo corpus sono stati ricavati mediante la risorsa Reverso Context² .

2 I rispettivi siti web dei corpora sono riportati nella sezione "corpora" della bibliografia finale. 
Il lavoro risulta articolato come segue: in $\S 2$ viene brevemente introdotta la nozione di deissi e il concetto centrale di centro deittico $(\S 2.1)$, che risulta molto utile come parametro di riferimento per l'individuazione di asimmetrie tra le due lingue. In $\S 3$ viene descritta più nel dettaglio la metodologia di raccolta dei dati alla base dell'approccio contrastivo utilizzato, nonché viene proposta una classificazione dei principali tipi di asimmetria individuati, poi illustrati ed esemplificati nei § 4-7. Infine, il lavoro si conclude con una sintesi dei vari livelli sui quali si articola la complessità delle asimmetrie nella codifica del riferimento deittico, soprattutto nella prospettiva delle difficoltà che esse comportano per gli apprendenti di LS o L2, data la non corrispondenza di forme e funzioni fra le due lingue.

\section{LA NOZIONE DI DEISSI}

Riportiamo qui e discutiamo brevemente le definizioni di deissi proposte rispettivamente per l'italiano dalla Grande Grammatica di Consultazione (2001) e per il russo da Grenoble (1998), che ha dedicato al tema diversi studi, tra cui una ricchissima monografia. Tali definizioni ci aiutano ad orientarci all'interno del fenomeno, prima di passare ad introdurre gli aspetti più specifici connessi alle asimmetrie deittiche:

Per «deissi» si intende $[\ldots]$ quel fenomeno linguistico per cui determinate espressioni richiedono, per essere interpretate, la conoscenza di particolari coordinate contestuali che sono l'identità dei partecipanti all'atto comunicativo e la loro collocazione spazio-temporale. (Renzi, Salvi \& Cardinaletti, 2001, III, p. 262)

Deixis specifically has to do with the ways sentences are anchored to their contexts of utterance, including such information about the participants as their roles in the speech event, and their spatial, temporal and social locations. (Grenoble, 1998, pp. 365-366)

Le due definizioni, molto simili nella sostanza, individuano nella deissi quel meccanismo di costruzione del significato che permette di ancorare l'enunciato alla realtà extralinguistica prendendo a riferimento 
il parlante, il suo interlocutore, nonché il tempo e il luogo in cui l'enunciato viene prodotto (tempo dell'enunciato, o TE) e il tempo e il luogo dei fatti richiamati dall'enunciato (momento dell'azione, o TA) ${ }^{3}$. Inoltre, nella definizione offerta da Grenoble si fa anche riferimento alla nozione di deissi sociale, individuata da Fillmore (1975, p. 38) per indicare il modo in cui le marche pronominali vengono usate al fine di marcare la distanza sociale tra gli interlocutori.

Entrambe le definizioni non forniscono indicazioni specifiche rispetto ai mezzi linguistici con cui la deissi viene realizzata, facendo riferimento a "espressioni" linguistiche, $\mathrm{o}$, ancora più genericamente, al modo in cui le "frasi" sono ancorate al contesto in cui viene prodotto l'enunciato, senza indicare se il meccanismo si avvalga esclusivamente di mezzi lessicali o anche di altra natura. Questa scelta a noi pare particolarmente appropriata, dal momento che come vedremo, la deissi, pur realizzandosi prevalentemente con mezzi lessicali (parole deittiche quali i pronomi personali o gli avverbi spaziali), può essere espressa in modo più elusivo anche con mezzi grammaticali: si pensi ad esempio all'uso del trapassato prossimo, con il quale l'italiano rende l'anteriorità nel passato rispetto al TA; tale deissi temporale in russo non viene tradizionalmente codificata con mezzi grammaticali, dal momento che questa lingua non dispone di un sistema di tempi che permettano di distinguere i diversi gradi di anteriorità nel passato; in alcuni casi specifici, tuttavia,

3 Nel presente articolo si farà ricorso alle sigle TA e TE per indicare, rispettivamente, il Tempo dell'Azione e quello dell'Enunciazione. I due concetti, introdotti da Reichenbach (1947) mediante le formule Event time e Speech time, sono stati ripresi nella Grande Grammatica italiana di consultazione (2001) nelle abbreviazioni MA (Momento dell'Azione) e ME (Momento dell'Enunciato) (Renzi et al., 2001, II, p. 17-23); per ragioni di chiarezza nel presente lavoro si preferisce utilizzare la coordinata $\mathrm{T}$ per i diversi riferimenti temporali. Il concetto di Centro Deittico da noi usato (CD) sostituisce quello che Reichenbach chiamava Reference time (Momento di Riferimento, MR) e che P. M. Bertinetto ha successivamente scisso in Localizzazione Temporale (LT), che indica l'intervallo di tempo o il momento in cui ha luogo l'evento, e Momento di Riferimento propriamente detto, che indica l'istante in cui il risultato di un evento già compiuto è visto come tuttora rilevante dal locutore, ossia, in altri termini, esprime "il valore aspettuale di compiutezza" (Renzi et al., 2001, II, p. 19). Il CD primario coincide con il TE, altrimenti indicato da $\mathrm{T}_{0}$. 
per esempio con i verbi che descrivono azioni reversibili (obratimye dejstvija), l'aspetto imperfettivo può svolgere una funzione analoga a quella del trapassato prossimo italiano, dal momento che indica un'azione conclusa (otkryval 'aveva aperto'), il cui risultato è stato annullato da un'azione contraria, e quindi implicitamente anteriore a quella marcata dall'aspetto perfettivo risultativo (otkryl 'aprì') e collocata nel TA:

(1) Tom aprì il cassetto che aveva aperto la sera dell'arrivo di Grace. ${ }^{4}$ Tom otkryl malen'kij jaščiček, kotoryj on otkryval v tot večer, kogda pojavilas' Grèjs.

(InterCorp, sottotitoli)

Il concetto di deissi richiede l'introduzione della nozione complementare di "centro deittico" (CD), che costituisce la prospettiva di luogo, tempo e persona che va adottata per l'interpretazione degli elementi deittici dell'enunciato (zero-point, nella terminologia proposta da Lyons, 1977). Grenoble illustra la nozione come segue:

[...] the unmarked spatial zero-point is the speaker's location, and the temporal zero-point is the moment of utterance, and the person zero-point the speaker him/herself. Therefore [...] the unmarked deictic center is the speaker and the speaker's location at the moment of utterance. (1995, p. 366)

A volte l'interpretazione di parole o espressioni deittiche è frutto dell'interazione di più $\mathrm{CD}$, che richiedono al ricevente lo spostamento (shift) da un CD primario ad un CD secondario: in tal senso un esempio tipico è costituito dalla coppia di verbi deittici dell'italiano andare e venire che presentano una distribuzione complementare a seconda della

${ }^{4}$ Abbiamo convenzionalmente deciso di usare il corsivo per tutti gli esempi tratti da corpus parallelo, indipendentemente dalla direzionalità della traduzione e tenendo conto del fatto che sia la versione italiana che quella russa potrebbero costituire testo di arrivo di un originale prodotto in una terza lingua (prevalentemente inglese, soprattutto nel caso degli esempi tratti dal corpus di sottotitoli). Dopo l'esempio viene sempre segnalata la fonte da cui è tratto, in assenza di tale indicazione l'esempio costituisce un nostro adattamento da una fonte reale, in questo caso per la traduzione proposta si è scelto di utilizzare il testo tondo tra virgolette semplici. 
posizione del parlante nel TE (CD primario) rispetto alla posizione assunta dagli altri partecipanti nel TA (CD secondario), per cui si rimanda a $\S 4.4$.

\section{L'APPROCCIO CONTRASTIVO}

La pratica didattica (nel nostro caso l'insegnamento universitario del russo a classi di studenti italofoni, in cui sono presenti anche studenti russofoni apprendenti di italiano) ha messo in rilievo che alcuni degli errori più frequentemente prodotti dagli studenti di entrambe le lingue sono facilmente prevedibili se si tiene conto del modo in cui L1 e L2 differiscono nell'espressione della deissi spaziale, temporale e personale. L'iniziale intuizione ha trovato conferma nell'analisi degli errori rintracciati in un piccolo corpus acquisizionale raccolto presso l'università di Roma Tre nell'ambito di un progetto di tandem per il perfezionamento delle competenze linguistiche tra pari: il corpus è formato dalle produzioni scritte in L2 che 10 coppie di studenti italofoni e russofoni impegnati nei task previsti dal progetto (organizzazione di una gita o visita di un monumento, preparazione di una cena tipica, visione di un film in lingua straniera, preparazione di una mappa concettuale) hanno fornito al docente alla fine delle loro attività; ciascun task andava svolto due volte, una in russo e una in italiano, in modo da dare la possibilità a entrambi i partecipanti di esercitare la L2; i testi prodotti sono di tipo spontaneo, come chat o e-mail organizzative, oppure semiguidato, come brevi relazioni sull'attività svolta.

Ai fini del presente lavoro abbiamo considerato gli errori prodotti dagli apprendenti russofoni che rivelano una diversa concettualizzazione del riferimento deittico tra L1 e L2, come nel seguente esempio:

(2) *Tra certo periodo tra Elena e Antonio erutta la passione e l'attrazione reciproca. (Tandem)

in cui si osserva l'estensione del deittico temporale tra ad un contesto proprio del deittico dopo (§ 4.1). Tale approccio ci ha permesso di identificare le forme di asimmetria più complesse per l'apprendente, che per 
chiarezza di esposizione sono state raggruppate, in base a criteri strutturali e funzionali, nei seguenti tipi:

a) ipercodifica $v s$ ipocodifica: rientrano in questa classe quei casi in cui una delle due lingue presenta una distinzione deittica assente nell'altra, oppure tende ad articolare maggiormente tale distinzione sul piano concettuale e di conseguenza formale; questa asimmetria costituisce un ostacolo per l'apprendente che deve imparare ad associare ad una forma della propria L1 due o più forme complementari della L2;

b) diversa articolazione interna di un analogo "spazio-tempo" di significazione deittica: in questo caso le due lingue possono presentare un'analoga complessità sul piano formale, tuttavia la distribuzione dei deittici impedisce di stabilire corrispondenze biunivoche tra L1 ed L2, l'apprendente pertanto non può sfruttare fenomeni di transfer positivo dalla propria L1;

c) adozione di una diversa prospettiva nella concettualizzazione del riferimento deittico: in questo caso le due lingue adottano differenti CD per designare un medesimo significato; fenomeni di transfer dalla propria L1 tendono a produrre realizzazioni agrammaticali o quantomeno marcate.

L'assegnazione delle diverse forme di asimmetria ad uno dei tipi sopra individuati non è rigida e ha pure finalità espositive: per esempio, il ricorso in russo alla prefissazione verbale, che permette di orientare il CD sia sul mittente (o source: VYslat' [elativo-inviare] / OToslat' [ABLATIVO-inviare]) che sul ricevente (o goal: PRIslat' [ALLATIVO-inviare]), è stato analizzato come un caso di ipercodifica del russo rispetto all'italiano, dal momento che nessuno degli equivalenti individuati in questa lingua (inviare, spedire, mandare) è esplicitamente orientato sul ricevente (v. § 4.4). Tuttavia sarebbe stato possibile anche attribuire il fenomeno alla classe (c), giacché l'uso della prefissazione in russo può implicare l'adozione di una diversa prospettiva nella codifica di un medesimo significato proposizionale. 


\section{IPERCODIFICA VS IPOCODIFICA}

In questa classe rientra la maggior parte dei fenomeni individuati, i quali possono manifestarsi in entrambe le lingue a livello lessicale (it. tra / dopo vs rus. čerez; it. prossimo / successivo vs rus. sledujuščij, it. già / ormai vs rus. uže, it. andare / venire vs rus pojti), grammaticale (in italiano esistono due forme di condizionale, presente e passato, mentre in russo tale distinzione è assente e un'unica forma copre entrambi i contesti dell'italiano), oppure nel ricorso ai diversi livelli del sistema (si pensi ai significati deittici di tipo aspettuale e azionale che vengono codificati in russo con mezzi morfologici e in italiano con mezzi lessicali).

\subsection{Distinzioni lessicali: tra e dopo vs čerez}

In italiano esistono due diverse preposizioni di tempo (tra e dopo) che permettono entrambe di individuare un riferimento futuro; tra rimanda a un momento successivo al TE, mentre dopo rimanda a un momento successivo al TA. TE e TA costituiscono rispettivamente il CD primario e secondario degli enunciati introdotti da tra e dopo. In russo tale distinzione, che pertiene alla deissi temporale, è assente, entrambe le preposizioni trovano un equivalente in čerez, che si limita ad introdurre un riferimento temporale futuro sottospecificato rispetto alla distinzione tra TE e TA, come mostrato dagli esempi che seguono:

(3) Dovrei essere lì tra due minuti.

Budu na meste čerez dve minuty.

(InterCorp, sottotitoli)

(4) $[. .$.$] e dopo due sole ore di sonno, la crisi di Max svanì lentamente [. .$.

[...] i čerez dva časa sna, krizis otstupil [...]

(InterCorp, sottotitoli)

Tale distinzione risulta particolarmente complessa per l'apprendente russofono, che tende a estendere una delle due preposizioni ai contesti dell'altra, come già mostrato nell'esempio (2) e in (5), dove *tra va a occupare i contesti di dopo: 
(5) *Tra certo tempo i ragazzi incontrano per caso ed insieme costruiscono i piani per la vita e vanno avanti. (Tandem)

Il quadro delle corrispondenze viene ulteriormente complicato da una terza preposizione dell'italiano, entro, che concettualizza il riferimento temporale successivo $\mathrm{T}_{1}$ come il limite massimo entro cui deve verificarsi un evento. Diversamente dalle precedenti, questa preposizione è indifferente al $\mathrm{CD}$, che può coincidere sia con il TE (6) che con il TA (7):

(6) Allora ti dico questo: voglio quel rapporto entro un'ora. Togda ja skažu tak: otčet dolžen byt' u menja v rukach v tečenie časa. (InterCorp, S. Larsson)

(7) Obama promise, in centinaia di discorsi ed interviste, che entro 6 mesi dalla sua elezione avrebbe riportato a casa le nostre truppe dall'Iraq. Obama zaverjal v sotnjach rečej i interv'ju, čto v tečenie šesti mesjacev posle ego izbranija, on otpravit domoj naši vojska $v$ Irake. (InterCorp, sottotitoli)

In entrambi gli esempi entro presenta come equivalente in russo la preposizione complessa $v$ tečenie lett. 'nel corso (di)', che concettualizza $\mathrm{T}_{1}$ non come un limite temporale, bensì come una durata, un lasso di tempo all'interno del quale deve necessariamente verificarsi l'evento di natura puntuale (l'arrivo del rapporto in (6), il rientro delle truppe dall'Iraq in (7)). La differenza alla base del modo in cui le due lingue rappresentano $\mathrm{T}_{1}$ (limite $v s$ durata), interferisce con la possibilità per l'apprendente di stabilire corrispondenze biunivoche tra le due lingue nella realizzazione di tale significato.

La preposizione $v$ tečenie, infatti, può essere utilizzata in russo anche per introdurre una durata con verbi non puntuali, realizzando così il suo significato letterale ('nel corso di / per (X tempo)'), che in italiano risulta ben distinto da quello di 'entro': 
(8) Klinton byl, požaluj, samym blizkim ego soratnikom v tečenie dvadcati let.

Clinton era stato forse il suo più stretto collaboratore per vent' anni. (InterCorp, S. Larsson)

4.2. Distinzioni lessicali: prossimo e successivo / seguente vs sledujuščij

Una distinzione analoga a quella descritta nel paragrafo precedente si presenta anche nel caso degli aggettivi dell'italiano prossimo e successivo / seguente: tutti qualificano un evento come posteriore, il primo in relazione al TE (9), i secondi due in relazione al TA (10); TE e TA costituiscono rispettivamente il CD primario e secondario dei relativi enunciati:

(9) Fino al prossimo plenilunio nessuno inquieterà il professore.

Do sledujuščego polnolunija professora ne potrevožit nikto.(InterCorp,

M. Bulgakov)

(10) La mattina seguente c'era silenzio.

Utro sledujuščego dnja bylo tichim. (NKRJA, V. Pelevin)

Come mostrano le versioni russe del testo, in questa lingua è assente la distinzione deittica spazio-temporale tra TE e TA, e un unico aggettivo (sledujuščij) viene usato per rimandare a un oggetto o a un evento collocato "dopo" nello spazio o nel tempo. L'assenza di tale distinzione si manifesta nelle produzioni degli apprendenti russofoni, che mostrano difficoltà a gestire l'ipercodifica dei riferimenti deittici dell'italiano. In (11) la studentessa, nella relazione relativa al task "visita a Castel Sant'Angelo", si riferisce alla trasformazione del castello in fortezza avvenuta nel periodo successivo o seguente usando l'espressione *prossimo periodo:

(11) Il *prossimo periodo castello è diventato un bastione fortificato per proteggere l'entrata nella città dai saccheggi. (Tandem) 


\subsection{Distinzioni lessicali: già e ormai vs uže}

In italiano esistono due avverbi, ormai e già, che possono essere utilizzati in modo piuttosto intercambiabile per individuare un riferimento temporale precedente ad un altro in cui ha luogo un cambiamento nello stato delle cose: il riferimento temporale può precedere il $\mathrm{TE}\left(=\mathrm{T}_{0}\right)$, come in (12), e venire segnalato da $\mathrm{T}_{-1}$, oppure precedere il TA: se il TA $\left(=\mathrm{T}_{-1}\right)$ a sua volta precede il $\mathrm{TE}$, tale riferimento temporale verrà segnalato da $\mathrm{T}_{-2}$ e indicherà antecedenza nel passato, come in (13), se invece il TA $\left(=\mathrm{T}_{2}\right)$ segue il $\mathrm{TE}$, tale riferimento temporale verrà segnalato da $\mathrm{T}_{1} \mathrm{e}$ indicherà un momento successivo a quello dell'enunciato, ma antecedente a quello degli eventi futuri di cui si sta parlando, come in (14):

(12) Mario è già / ormai arrivato a Mosca $\mathrm{T}_{-1}=\mathrm{T}_{0}(=\mathrm{TE}) \rightarrow$ in $\mathrm{T}_{-1}$ Mario arriva a Mosca dove è ancora presente al TE

(13) Mario era già / ormai arrivato a Mosca, quando ti ho telefonato $\mathrm{T}_{-2} \mathrm{~T}_{-1}(=\mathrm{TA}) \quad \mathrm{T}_{0}(=\mathrm{TE}) \rightarrow$ in $\mathrm{T}_{-2}$ Mario arriva a Mosca dove si trova ancora nel TA (momento della telefonata), che precede il TE

(14) Per le sette di sera Mario sarà già / ormai arrivato a Mosca $\mathrm{T}_{0}(=\mathrm{TE}) \_\mathrm{T}_{1}=\mathrm{T}_{2}(=\mathrm{TA}) \rightarrow$ Mario arriverà a Mosca nel momento in $\mathrm{T}_{1}$ che precede il TA (le sette di sera)

La distinzione tra le due forme non si associa pertanto alla codifica di diversi CD (visto che entrambi gli avverbi segnalano genericamente anteriorità sia rispetto al TE che al TA), ma si realizza sul piano implicazionale: già e ormai presuppongono infatti un differente atteggiamento del parlante in relazione al cambiamento prodotto nello stato delle cose, come illustrato in $(15 \mathrm{a}-\mathrm{b})$ :

(15) a. I bambini si sono già svegliati

'Deti uže prosnulis'"

b. I bambini si sono ormai svegliati

'Deti uže prosnulis'"

In (15a) viene semplicemente costatato un fatto: nel momento $T_{-1}$ che precede il momento dell'enunciato, i bambini si sono svegliati; in 
(15b) invece, al precedente significato si aggiunge un'informazione di tipo implicito relativa al fatto che il risveglio dei bambini viene percepito come irreversibile e che i partecipanti all'evento descritto sono rassegnati o infastiditi ${ }^{5}$. In russo uže funziona da equivalente per entrambi gli avverbi, lasciando al ricevente il compito di aggiungere al suo significato eventuali inferenze relative all'irreversibilità dell'evento e al fastidio provocato; in altre parole, tale informazione in russo non viene esclusa, ma deve essere ricavata sulla base di conoscenze contestuali (si tratta di bambini che dormono poco e avrebbero necessità di dormire più a lungo) $\mathrm{o}$ enciclopediche (i bambini piccoli in genere sono vivaci e richiedono attenzioni).

A questa differenza appena discussa, se ne associano altre che agiscono sempre sul piano delle implicazioni: già infatti implica la natura repentina e precoce dell'evento che ha luogo in $\mathrm{T}_{-1}$ (Bertinetto, 1994, cit. in Manzotti \& Zampese, 2010, p. 8).

In (16), ad esempio, già comunica che la rottura della lampada non rientra nel normale svolgersi degli eventi (solitamente non ci si aspetta che un bambino possa provocare un simile danno nel breve tempo che intercorre dal suo arrivo), ma suggerisce tuttavia che l'incidente era in qualche modo inevitabile se associato agli schemi comportamentali di quel determinato bambino (che magari è particolarmente distratto o vivace), verso il quale il parlante nutre chiaramente pregiudizio. Tra le inferenze suggerite dal già vi è pertanto quella relativa all'irritazione nel parlante nei confronti dell'evento e dell'agente:

${ }^{5}$ Questa differenza diventa esplicita negli usi olofrastici di già e ormai come replica ad una precedente asserzione. L'uso di già indica che si concorda con quanto asserito dall'interlocutore ed è parafrasabile con infatti; in maniera analoga, la forma funziona anche come segnale fatico di ricezione: - Deve essere stata dura. / - Già, non abbiamo mai avuto una vita facile (InterCorp, sottotitoli); l'uso di ormai invece implica rassegnata accettazione per il corso non propizio degli eventi ed è solitamente accompagnato nella pronuncia da un prolungamento della sillaba tonica e un'intonazione ascendente: - Che fai qui? / - Sono tornata da te. / - Non dovevi venire qui. / -Ormai... (InterCorp, sottotitoli). 
(16) È qui da due ore e ha già rotto una lampada.

On byl zdes' dva časa, a on uže razbil lampu.

(Reverso, sottotitoli)

In questo contesto già è solo parzialmente sostituibile con ormai, che sposterebbe l'implicazione in direzione dell'irreversibilità dell'evento e del dispiacere del parlante, ma risulterebbe poco coerente con l'affermazione che precede (è qui da due ore), la quale sottolinea la rapidità con cui l'incidente ha avuto luogo: non a caso, in simili contesti, il circostanziale di tempo è spesso associato a un focalizzatore (soltanto / solo / unicamente da due ore e già...).

Infine, già può implicare anche il carattere reiterato dell'evento: in (17) l'uso dell'avverbio lascia inferire a chi ascolta che il parlante abbia di nuovo perso il portafogli o qualche altro oggetto personale:

(17) [...] ho già perso il portafoglio una volta e...

Ponimaete, ja na dnjach uže terjal košelek...

(Reverso, sottotitoli)

In questo contesto già non può essere sostituito da ormai, che implicando l'irreversibilità dell'evento non è compatibile con una lettura reiterata dello stesso.

Come si può vedere dagli esempi, sia in (16) che in (17) la versione russa del testo evidenza di nuovo un'equivalenza tra già e uže. A tale proposito, bisogna aggiungere che l'uso dell'aspetto imperfettivo con il verbo terjat' 'perdere', oltre ad essere inteso in senso puramente esperienziale $^{6}$, potrebbe implicare l'annullamento del risultato dell'azione (e quindi il ritrovamento del portafogli): la possibilità di codificare con mezzi aspettuali un'informazione che in italiano rimarrebbe implicita, riguarda la specifica classe semantica dei verbi "reversibili", in ogni

6 Con il termine "esperienziale" si intende qui l'uso generico dell'imperfettivo per nominare un'azione che ha avuto luogo nel passato. A tale uso dell'imperfettivo si fa solitamente riferimento negli studi aspettologici russi con l'etichetta di "significato generico-fattuale" (obščefaktičeskoe značenie), che tuttavia in questo lavoro preferiamo non adottare. 
caso tale interpretazione per attivarsi necessita anche in russo di un contesto adeguato, come potrebbe essere quello appena presentato.

\subsection{Distinzioni lessicali: andare e venire vs idti, chodit', pojti}

In italiano i verbi andare e venire descrivono entrambi movimento verso una destinazione o un obiettivo ( $\mathrm{goal}$ ), ma differiscono in alcune implicazioni relative al CD secondario, ovvero il luogo in cui si troveranno gli interlocutori - parlante, ascoltatore e soggetto dell'enunciato (nel caso in cui quest'ultimo non coincida con i primi due) - nel TA, come mostrato in (18):

(18) a. Stasera ti va di andare alla partita?

'Ne chočeš' pojti večerom na igru?'

b. Stasera ti va di venire alla partita?

'Ne chočeš' pojti večerom na igru?'

In (18a) il parlante chiede al proprio interlocutore se la sera si recherà alla partita: 1'uso di andare non fornisce informazioni sulle intenzioni del parlante per la serata, invece l'uso di venire in (18b) implica che anche il parlante si recherà a vedere la partita; venire infatti attiva un CD secondario, che coincide con il luogo (probabilmente uno stadio) in cui si troverà il parlante nel TA (cioè la sera): assumendo la prospettiva del CD secondario, lo spostamento dell'interlocutore viene visto come un movimento diretto verso il parlante, ovvero è come se il parlante, nel momento in cui formula il suo enunciato, si proiettasse idealmente già allo stadio e chiedesse all'interlocutore se è intenzionato a raggiungerlo lì (anche se poi sul piano della realtà i due potrebbero recarsi allo stadio insieme). Sebbene la versione in (18a), come già detto, non implichi l'assenza del parlante nel TA, il fatto che l'italiano disponga di una forma per segnalarne appositamente la presenza, spinge ad attribuire all'uso di andare un significato opposto a quello di venire per quanto concerne l'attivazione di riferimenti deittici secondari. Nella letteratura sulla deissi si sostiene che i verbi del tipo andare siano orientati sul punto di partenza (source-oriented), mentre quelli del tipo venire sul punto di arrivo (goal-oriented). In russo la differenza di prospettiva deittica as- 
sociata all'opposizione andare / venire non viene codificata sul verbo; le traduzioni a (18a-b) mostrano infatti che una medesima forma verbale (pojti) riesce a coprire la portata semantica dei due verbi dell'italiano. In russo va detto tuttavia che esistono altri possibili equivalenti per la coppia di verbi italiani: al verbo perfettivo pojti si affiancano infatti l'imperfettivo unidirezionale idti e l'imperfettivo multidirezionale chodit'. Tale distinzione, che riguarda essenzialmente le caratteristiche azionali dei verbi di movimento in russo e coinvolge fenomeni di deissi spaziotemporale, verrà approfondita nel prossimo paragrafo.

Per i motivi appena esposti, l'opposizione tra andare e venire costituisce per l'apprendente russofono un evidente ostacolo ${ }^{7}$. L'esempio (19), tratto da una conversazione via chat tra L (studentessa italofona) e A (studentessa russofona), relativa al task "organizzazione di una cena tipica italiana", evidenzia in L consapevolezza metalinguistica del problema costituito dai verbi di moto in entrambe le lingue:

(19) L: Ma vieni in metro o in autobus?

A: Mi sa che vado con la metro, con autobus a Roma nn si sa mai

L: Ok! Comunque in questo caso bisogna utilizzare il verbo venire

Vengo con la metro). I verbi di moto sono un casino ahah io quando li so in russo faccio sempre molta fatica ad esempio (:) (Tandem)

L si informa da A sul mezzo utilizzato per venire da lei: l'uso del presente (con valore di futuro) è giustificato dal fatto che lo spostamento è diretto verso il parlante stesso, il quale nel TA si troverà nello stesso luogo di A. La risposta di A ( ${ }^{*}$ vado con la metro), corretta poi da L, per quanto grammaticale, risulta inadeguata, perché implicherebbe che il luogo verso cui A è diretto non è quello in cui si trova L.

7 Per una discussione più approfondita sull'argomento si rimanda a Lewandowski, che utilizza l'approccio contrastivo per dimostrare come tali asimmetrie nella codifica del riferimento deittico costituiscano una difficoltà anche per apprendenti polacchi di spagnolo. Analogamente all'italiano anche lo spagnolo codifica sul verbo "the speaker's location at the reference time" (Lewandowski, 2014, p. 46). 


\subsection{Distinzioni lessicali: idti, chodit' vs andare}

Al verbo italiano andare, com'è noto, corrispondono più verbi di movimento in russo. Questi si distinguono, oltre che per il modo del movimento (a piedi, con mezzo, di corsa ecc.) e per l'ambiente in cui esso avviene (su terra, in aria, in acqua), anche per le proprietà azionali, su cui si basano la suddivisione in verbi di moto determinato e indeterminato e i fenomeni di deissi spazio-temporale che li riguardano.

Se consideriamo, per esempio, i due verbi chodit' / idti, che corrispondono in italiano ai verbi andare / venire (a piedi), l'evento di moto descritto dal primo ha le caratteristiche dell'azione abituale o semplicemente reiterata nel tempo, mentre il secondo si riferisce di norma a un'azione singola e contestualizzata. In letteratura al caso di chodit' è di solito associata l'espressione di neaktual'noe značenie (significato usuale), mentre idti rappresenta un caso di aktual'noe značenie (significato processuale).

Il seguente esempio illustra il primo dei due casi:

(20) Levka chodit v detskij sad (NKRJa, S. Dovlatov)

Levka va all'asilo

Nell'esempio il significato azionale del verbo chodit', che descrive un movimento multiplo sul piano spazio-temporale, implica l'assenza di un unico $\mathrm{CD}$ temporale in relazione a $\mathrm{T}_{0}(=\mathrm{TE})$. Il secondo caso, al contrario, riguarda un movimento singolo in una direzione, descritto nel suo svolgersi, come si vede nell'esempio che segue:

\section{(21) Chozjain vstaet, idet $k$ vešalke (NKRJa, V. Erofeev) Il padrone si alza in piedi, va verso l'attaccapanni}

In questo caso l'uso del presente narrativo di idti comporta che il CD interno alla narrazione coincida sul piano temporale con $T_{-1}$ $(=\mathrm{TA})$, distinto da $\mathrm{T}_{0}(=\mathrm{TE})$. Qualora invece il verbo sia usato al presente per descrivere un evento che si svolge alla presenza di chi lo racconta, come può accadere nel corso di una cronaca sportiva, non ci sarebbe distinzione fra $\mathrm{T}_{-1}$ e $\mathrm{T}_{0}(\mathrm{TA}=\mathrm{TE})$. In italiano, com'è evidente, 
le distinzioni considerate in relazione ai lessemi verbali russi chodit' / idti non emergono. Per questo, nel caso considerato di "azione singola, contestualizzata $v s$ azione reiterata, abituale", appare legittimo attribuire all'italiano un comportamento di ipocodifica del riferimento deittico spazio-temporale e al russo, invece, un comportamento di ipercodifica.

4.6. Distinzione grammaticale: condizionale presente e passato $v s$ condizionale by

Un fenomeno di ipercodifica $v s$ ipocodifica basato sul ricorso a categorie grammaticali è rilevato nella diversa strutturazione del modo condizionale nelle due lingue. In italiano l'uso del condizionale presente denota un rapporto di identità fra $\mathrm{CD}$ e TE, com'è evidente nell'esempio che segue:

(22) Potresti dirmi la verità

Ty mog by skazat' mne pravdu

(Reverso, sottotitoli)

Dal punto di vista pragmatico gli enunciati di entrambe le lingue hanno il valore di un'esortazione o di un consiglio nell'ambito di un'interazione informale tra pari. Qualora, però, il presente condizionale sia sostituito in italiano dal passato dello stesso modo, il CD non coincide con TE, ma con TA. In tal caso, l'atto linguistico viene percepito come un rimprovero:

(23) Avresti dovuto dire la verità Ty mog by skazat' pravdu

(Reverso, sottotitoli)

Gli esempi mostrano che, mentre in italiano si riscontra una specifica codifica per ciascuno dei due atti linguistici, mediante l'uso del condizionale presente o passato, in russo si ha una medesima struttura di predicato che determina, insieme all'ipocodifica del riferimento deittico temporale, anche l'impossibilità di definire la forza illocutiva all'enunciato fuori dal contesto. 
4.7. Morfologia vs lessico: PRIslat' / VYslat' / OToslat' vs mandare

Tra i fenomeni che rientrano nell'asimmetria del tipo "ipercodifica vs ipocodifica" (v. punto (a), § 3), vi è l'uso dei prefissi spaziali in russo, che uniti ad una medesima base verbale permettono di formare derivati che si differenziano sul piano semantico esclusivamente per l'adozione di un differente $\mathrm{CD}$. L'italiano, come già accennato in $\S 3$, pur disponendo di mezzi morfologici derivazionali per codificare la deissi spaziale, non ne fa un uso sistematico ${ }^{8}$. Se consideriamo, per esempio, il gruppo di verbi con base -slat', a cui si può genericamente associare il significato di 'inviare, mandare', osserviamo che i diversi prefissi spaziali che ad essa si uniscono (pri- [allativo], vy- [elativo], ot- [ABLATIVo]), permettono di orientare il movimento sul ricevente o sul mittente, come illustrato negli esempi che seguono:

(24) Potom prislali nam četyre buterbroda

Ci hanno mandato quattro panini (NKRJa, A. Camilleri)

(25) Zvonite sejčas $v$ miliciju, čtoby vyslali pjat' motocikletov (NKRJa, M. Bulgakov)

Telefonate subito alla polizia perché mandino cinque moto

In (24) il CD è rappresentato, mediante il prefisso pri-, dal Beneficiario (coloro che ricevono i panini), e dal tempo e luogo della ricezione. In (25), invece, il prefisso verbale $v y$ - sposta il CD sul Benefattore (la polizia, che invia le motociclette), e sul tempo e luogo dell'invio. In italiano, in (24) come in (25), si ricorre all'uso del medesimo lessema verbale (mandare), e a orientare il CD sul parlante-ricevente provvede in (24) il pronome obliquo $c i$ 'a noi'. Ciò significa che in russo la deissi di persona (beneficiario / goal vs benefattore / source) e quella spaziotemporale (tempo e luogo in cui ha inizio l'azione $v s$ tempo e luogo in cui si conclude l'azione) sono codificate sul verbo mediante i prefissi verbali, mentre in italiano sono esplicitate dalla presenza di eventuali

${ }_{8}$ Per esempio, a partire dalla base portare, vengono formati i verbi deittici esportare e importare, ma gli stessi prefissi spaziali non si applicano alle basi spedire o mandare. 
elementi argomentali come il complemento di termine, dal momento che il significato lessicale del verbo non è deitticamente orientato.

\section{DIVERSA STRUTTURAZIONE DEL RIFERIMENTO DEITTICO}

Le due lingue ricorrono a risorse grammaticali differenti per la codifica del riferimento deittico (v. punto (b), § 3), pertanto possono presentarsi analogamente complesse sul piano strutturale, ma l'impiego di diverse categorie grammaticali rende difficoltosa per l'apprendente l'individuazione di corrispondenze biunivoche. Un caso è rappresentato dalla consecutio temporum, dove il CD collocato nel futuro rispetto a TA prevede, in italiano, l'uso delle categorie di tempo e modo del verbo, mentre il russo si serve della sola categoria temporale. Nell'esempio che segue TA e TE non coincidono e il CD è futuro rispetto al TA:

\section{(26) Ha detto che sarebbe venuto}

'On skazal, čto pridet'

In tal caso l'italiano ricorre al modo condizionale e al tempo passato (condizionale composto) del verbo venire. In russo, invece, il modo del verbo rimane l'indicativo e il riferimento deittico è ancorato al tempo futuro del verbo.

Nel caso invece in cui TA e TE coincidano (nel presente) e CD sia nel futuro, in entrambe le lingue la codifica di CD è affidata alla categoria temporale del verbo:

\section{(27) Dice che verrà \\ 'On govorit, čto pridet'}

Nella consecutio temporum è quindi l'italiano a mostrare ipercodifica nell'uso delle categorie verbali in relazione al riferimento deittico temporale, mentre il russo, dove il futuro indica posteriorità in assoluto (indipendentemente da TE e TA), presenta un fenomeno di ipocodifica.

Un altro caso di asimmetria delle due lingue nell'uso dei mezzi grammaticali per l'espressione della deissi si registra nella contrappo- 
sizione fra tempo e aspetto. Com'è noto, in italiano, e in generale nelle lingue romanze, sono i tempi verbali che veicolano il complesso sistema dei significati aspettuali del verbo (Renzi, Salvi \& Cardinaletti, 2001, II, pp. 41-62). Nel particolare caso dei verbi "reversibili", il cui significato lessicale può essere associato al suo contrario (come, per esempio, aprire / chiudere), l'aspetto imperfettivo può implicare in russo che l'azione intrapresa nel momento $\mathrm{T}_{-2}$ abbia annullato il suo risultato nel momento $\mathrm{T}_{-1}$ anteriore a $\mathrm{TE}=\mathrm{T}_{0}$ :

$$
\mathrm{T}_{-2} \rightarrow \quad \leftarrow \mathrm{T}_{-1} \text {---------------- } \mathrm{T}_{0}(=\mathrm{TE})
$$

In italiano tale informazione implicita è solitamente ricavabile dal contesto, anche se può essere espressa in modo più esplicito mediante l'uso del trapassato prossimo in alternativa al passato prossimo.

In (28) l'aspetto imperfettivo implica in russo la completa anteriorità dell'evento (arrivo dell'amico in $\mathrm{T}_{-2}+$ permanenza + partenza in $\mathrm{T}_{-1}$ ) rispetto a $\mathrm{T}_{0}(=\mathrm{TE})$; in italiano, invece, l'informazione relativa alla ripartenza dell'amico è ricavabile esclusivamente dal contesto:

(28) Prichodil tvoj drug, ostavil zapisku dlja tebja

'È venuto il tuo amico, ha lasciato un biglietto per te'

In (29), invece, la completa anteriorità dell'evento (apertura della finestra e sua successiva chiusura) rispetto a TE è espressa anche in italiano con mezzi grammaticali, mediante l'uso del trapassato prossimo (sebbene anche in tale contesto sarebbe stato pienamente ammissibile l'uso del passato prossimo):

(29) Kto otkryval okno?

'Chi aveva aperto la finestra?'

\section{ASIMMETRIE PROSPETTICHE}

Fra $\mathrm{i}$ casi in cui le due lingue adottano diverse prospettive nella concettualizzazione del riferimento deittico (v. punto (c), § 3), si contano fenomeni di tipo lessicale, come i predicati conversivi, e di tipo gram- 
maticale, come la possibilità di concettualizzare un evento tanto come concluso che come ripetuto o durativo.

\subsection{I predicati conversivi: \\ prendere vs polučit', ricevere vs prinjat'}

Consideriamo i predicati prendere (it) vs polučit' (ru). I due verbi appartengono al gruppo dei cosiddetti predicati conversivi e assumono nelle due lingue prospettive diverse nella descrizione del medesimo frame.

Nel seguente esempio:

(30) Mi piacerebbe prendere la patente Ja by chotela polučit' prava (Reverso, sottotitoli)

in italiano il CD è quello del soggetto Agente, ossia colui che "prende" la patente, mentre in russo quello del soggetto Beneficiario, colui che "riceve" la patente. In quest'ultimo caso, inoltre, il predicato rimanda alla presenza implicita del Benefattore o source, ossia l'istituzione che rilascia il documento.

I predicati conversivi prevedono anche la possibilità di un rovesciamento del CD. Nello specifico, ne è un esempio la coppia di verbi ricevere vs prinjat', come risulta dagli enunciati che seguono:

(31) È solo che non ero affatto pronta a ricevere ospiti Ja prosto, ponimaete, ne gotova poka prinimat' gostej (Reverso, sottotitoli)

Mentre in italiano il CD è costituito dal soggetto Beneficiario, ossia colui che "riceve" gli ospiti, con la presenza implicita di un luogo (source) da cui gli ospiti si muovono, in russo esso è rappresentato dal soggetto Agente, ovvero colui che accoglie gli ospiti.

\subsection{Concettualizzazione di un evento: concluso $v s$ ripetuto o durativo}

Sul piano grammaticale, il fenomeno della diversa prospettiva nella descrizione dell'evento in riferimento al CD si registra, per esempio, 
nei predicati con il passato prossimo in italiano $v s$ i predicati di aspetto imperfettivo in russo per la descrizione di eventi concettualizzati nelle rispettive lingue come conclusi e reiterati o durativi. Per quanto riguarda il russo, viene qui introdotta la categoria dell'aspetto verbale e il suo uso deittico. Com'è noto, i significati primari degli aspetti perfettivo e imperfettivo sono deittici, in quanto gli eventi descritti sono localizzati sull'asse temporale con riferimento al TE.

Nel seguente esempio:

(32) Ne raz mne prichodilos' slyšat' pochvaly $v$ adres otca (NKRJa, V. Erofeev)

Più volte mi è capitato di sentire elogiare mio padre

il medesimo evento è visualizzato secondo prospettive differenti, espresse in italiano dal passato prossimo del verbo (è capitato) e in russo dall'aspetto imperfettivo (prichodilos'). In italiano l'evento reiterato nel lasso di tempo fra $\mathrm{T}_{-1}$ e $\mathrm{T}_{0}$ è descritto dal passato prossimo come compiuto, chiuso rispetto a $\mathrm{T}_{0}(=\mathrm{TE})$. In russo, invece, esso è descritto da un verbo di aspetto imperfettivo e viene visualizzato sull'asse temporale come reiterato un numero $n$ di volte, in un segmento temporale aperto fra $\mathrm{T}_{-n} \mathrm{e} \mathrm{T}_{0}(=\mathrm{TE})$.

Lo stesso accade nel caso dei predicati con significato azionale durativo. Ne sono un esempio i seguenti enunciati, che come i precedenti presentano una forma di passato prossimo in italiano e una di aspetto imperfettivo in russo:

(33) Etot gospodin echal za nami ves' den' (NKRJa, V. Nabokov) Quella persona ci ha seguiti per tutto il giorno

Il passato prossimo dell'italiano ( $c$ i ha seguiti) esprime l'aspetto inclusivo (Salvi \& Vanelli, 2004, p. 115), dal momento che in $\mathrm{T}_{0}(=\mathrm{TE})$ l'evento durativo, che ha luogo fra $\mathrm{T}_{-1}$ e $\mathrm{T}_{0}$, è focalizzato come concluso. In russo, invece, l'aspetto imperfettivo del verbo (echal) mette a fuoco la durata del processo che si svolge nel lasso temporale (ves' den') fra $\mathrm{T}_{-1}$ e $\mathrm{T}_{0}(=\mathrm{TE})$. 
Come si è visto, il tempo verbale (passato prossimo) in italiano e l'aspetto imperfettivo in russo operano diversi agganci deittici per descrivere il medesimo evento sull'asse del tempo. Tale asimmetria nella rappresentazione di un evento passato come concluso in italiano e come prolungato in russo si riflette anche nelle produzioni dei russofoni partecipanti al progetto Tandem, che tendono a trasferire in italiano lo schema concettuale del russo. In (34) la studentessa riporta la ricetta della lasagna preparata per il task "organizzazione di una cena italiana":

(34) Abbiamo grattugiato i formagi. [...] Abbiamo messo dal'inizio un po' di ragu dentro. Dopo abbiamo distribuito la pasta, sotto abbiamo messo il ragu. Abbiamo aggiunto il formagio e abbiamo ripetto tutto 5 volte. Sopra abbiamo finito con il parmegiano. La taglia *era messa sul forno, per 15 minuti. (Tandem)

Il carattere sequenziale delle azioni compiute per preparare la ricetta viene correttamente reso dal passato prossimo, tuttavia per l'ultimo passaggio, quello della cottura della lasagna, l'informante preferisce l'uso di un imperfetto di forma passiva, ritenuto erroneamente più adeguato a rendere la durata della permanenza della teglia nel forno (per 15 minuti): tale uso risulta incompatibile in italiano con il carattere finito dell'evento. Analogamente, in (35), la partecipazione dei propri familiari alla Grande Guerra Patriottica richiederebbe il passato prossimo (hanno partecipato) in luogo dell'imperfetto ( *artecipavano), dal momento che la guerra costituisce un evento concluso:

(35) Il 9 maggio in Russia è una grande festa nazionale perché festeggiamo il Giorno della Vittoria nella Grande Guerra Patriottica. [...] Quasi ogni famiglia in Russia e tutta ex-Unione Sovietica ha famigliari che *partecipavano o che sono morti in questa guerra. (Tandem)

\section{TIPI DI ASIMMETRIA NELL'INTERFACCIA LESSICO-PRAGMATICA}

Un particolare tipo di asimmetria emerge nei casi in cui le due lingue dispongono di analoghi mezzi lessicali per codificare un medesimo riferimento deittico, tuttavia essi differiscono nel modo in cui questo si 
aggancia alla realtà extratestuale. Il mancato controllo di questo tipo di asimmetria produce fallimenti sul piano comunicativo, ovvero pragmatico, ma non errori lessicali o grammaticali.

Nell'esempio seguente:

(36) Sanitary otpravili ego na vtoroj etaž (NKRJa, A. Kurkov)

Gli infermieri lo mandarono al primo piano

il CD spaziale è identico nelle due frasi, ma i numerali ordinali con cui è indicato non sono equivalenti dal punto di vista lessicale: in russo il conteggio dei piani indica come primo piano quello che in italiano è indicato come piano terra. Per questo, in assenza di un'adeguata conoscenza da parte dell'interlocutore di tale asimmetria possono insorgere fraintendimenti sul piano comunicativo.

Un caso analogo è rappresentato dall'espressione con cui sono indicate le fermate dell'autobus successive a quella che segue immediatamente $\mathrm{T}_{0}(=\mathrm{TE})$ :

(37) Čerez odnu ostanovku Andrej vyšel (NKRJa, A. Gračev)

'Alla seconda fermata Andrej scese'

Nell'esempio il riferimento al CD spazio-temporale è affidato a mezzi lessicali che non ammettono un'interpretazione letterale in italiano: l'indicazione čerez odnu ostanovku (lett. 'tra una fermata') segnala di "attraversare" una fermata prima di scendere. Pertanto, diversamente da quanto suggerito letteralmente, la fermata a cui scendere è la seconda. L'assenza di specularità semantica tra le due lingue rende plausibile l'equivoco nello scambio comunicativo.

La stessa preposizione (čerez den' lett. 'tra un giorno') si trova nell'indicazione del CD temporale del seguente esempio:

(38) Čerez den' po restoranam chodim (NKRJa, S. Dovlatov)

Un giorno sì un giorno no andiamo al ristorante 
Anche in questo caso, per comprendere correttamente l'informazione trasmessa dall'enunciato è necessario conoscere i modi in cui le due lingue si servono del lessico per codificare il medesimo CD temporale.

\section{OSSERVAZIONI CONCLUSIVE}

La prassi didattica mette in luce alcune difficoltà per l'apprendente che, come osservato, possono essere previste e spiegate in termini di asimmetrie nella codifica della deissi. Tali asimmetrie si manifestano a vari livelli:

I. nella maggiore articolazione semantica e strutturale di un riferimento deittico (ipercodifica) in una lingua rispetto all'altra, in cui talune distinzioni possono essere assenti (ipocodifica): ciò comporta che non vi è corrispondenza biunivoca di forma e funzione tra le due lingue (\$ 4);

II. nella diversa articolazione interna di un medesimo segmento di significazione deittica: in questo caso le due lingue si presentano come analogamente complesse sul piano strutturale, ma ricorrono a risorse appartenenti a diversi livelli del sistema e anche in questo caso non è possibile stabilire corrispondenze esatte tra forme e funzioni di una lingua con l'altra (§ 5);

III. nel diverso orientamento del $\mathrm{CD}$, come ad esempio nei predicati conversivi (§ 6).

La deissi pertanto si manifesta come un fenomeno già di per sé complesso, talvolta ulteriormente complicato dall'interazione di diversi CD in uno stesso contesto (come nel caso della distinzione in italiano tra i verbi andare e venire). Per l'apprendente, l'uso degli indicatori deittici si traduce non solo in errori lessicali e grammaticali, ma anche in fallimenti sul piano pragmatico-comunicativo (§ 7).

In futuro sarebbe interessante poter ampliare l'analisi contrastiva finora condotta tra russo e italiano per quanto concerne la codifica della deissi, al fine di individuare e sistematizzare le asimmetrie anche in una prospettiva didattica. 


\section{BIBLIOGRAFIA}

Bertinetto, P.M. (1994). «Ormai». In P. Cipriano, P. di Giovine \& M. Mancini (Eds.), Miscellanea di studi linguistici in onore di Walter Belardi (pp. 789-910). Roma: Il Calamo.

Fillmore, C.J. (1975). Santa Cruz lectures on deixis: 1971. Bloomington, IN: Indiana University Linguistics Club.

Grenoble, L.A. (1995). Spatial configurations, deixis and apartment descriptions in Russian. Pragmatics, 5(3), 365-85.

Grenoble, L.A. (1998). Deixis and Information Packaging in Russian Discourse. Amsterdam / Philadelphia: John Benjamins Publishing Company.

Goddard, C., \& Wierzbicka, A. (2014). Words and Meanings: Lexical Semantics across Domains, Languages and Cultures. Oxford: Oxford University Press.

Lewandowski, W. (2014). Deictic Verbs: Typology, Thinking for Speaking and SLA. SKY Journal of Linguistics, 27, 43-65.

Lyons, J. (1977). Semantics. Vols 1 \& 2. Cambridge: Cambridge University Press.

Manzotti, E., \& Zampese, L. (2010). Un avverbio (a suo modo) scalare? Sulla semantica di it. or(a)mai. In P. Hadermann \& O. Inkova (Eds.), Approches de la scalarité, (pp. 3-40). Ginevra: Droz.

Reichenbach, H. (1947). Elements of Symbolic Logic. London: Mac Millan. Renzi, L., Salvi, G., \& Cardinaletti, A. (2001). Grande grammatica italiana di consultazione (2nd ed.). Bologna: Il Mulino.

\section{Corpora}

Corpus Nazionale di Lingua Russa: www.ruscorpora.ru

Corpus Nazionale di Lingua Ceca, Kontext:

www.kontext.korpus.cz, www.opensubtitles.com/it

Reverso Context: www.context.reverso.net

Riassunto: Il presente contributo si pone l'obiettivo di indagare, mediante un approccio contrastivo, alcuni casi di asimmetria tra l'italiano e il russo nella codifica del riferimento deittico. In relazione al concetto di centro deittico (zero-point, secondo la terminologia proposta da Lyons, 1977), sono analizzati alcuni esempi complessi di differente ancoraggio deittico nelle due lingue, raggruppati in base a criteri strutturali e funzionali: a) casi di ipercodifica $v s$ ipocodifica (come nell'uso di tra e dopo vs čerez), b) casi di diversa articolazione interna di un analogo riferimento deittico (come nell'uso di modo e tempo del verbo vs solo tempo nella consecutio temporum) e c) casi in cui il riferimento 
deittico viene concettualizzato secondo prospettive diverse (come nei predicati conversivi). Tali forme di asimmetria risultano particolarmente complesse per gli apprendenti delle due lingue, motivo per cui l'analisi degli errori prodotti nella codifica della deissi può rivelarsi un utile strumento anche in una prospettiva didattica.

Parole chiave: deissi, asimmetrie deittiche, apprendimento / acquisizione della seconda lingua, grammatica contrastiva, corpora paralleli 